\title{
COMPARISON AND APPLICATION OF TWO GREEN'S MATRICES
}

\section{A. L. DEAL, III}

1. Purpose. The purpose of this paper is to demonstrate a close but not obvious similarity between two Green's matrices due respectively to W. M. Whyburn and R. H. Cole and then to apply these matrices to the solution of a difference system.

2. Whyburn's Green's matrix. Whyburn [1] has given a Green's matrix,

$$
\begin{aligned}
G_{W}(x, t) & =U(x) H_{1}^{I}(U)\left[A U(a)+\int_{a}^{t} F(s) U(s) d s\right] U^{I}(t), \quad t<x \\
& =-U(x) H_{1}^{I}(U)\left[B U(b)+\int_{t}^{b} F(s) U(s) d s\right] U^{I}(t), \quad t>x
\end{aligned}
$$

for the system,

$$
\begin{aligned}
& L_{1}(Y)=Y^{\prime}(x)+P(x) Y(x)=0 \\
& H_{1}(Y)=A Y(a)+B Y(b)+\int_{a}^{b} F(x) Y(x) d x=0 .
\end{aligned}
$$

This Green's matrix yields a solution of the nonhomogeneous $L_{1}(Y)$ $=Q(x), H_{1}(Y)=D$, assuming $A, B, D$ constant $n \times n$ matrices and $P, Q, F$ matrices of Lebesgue summable functions. $U$ is nonsingular on $[a, b]$ and $U^{\prime}(x)+P(x) U(x)=0$. The superscript $I$ is used to indicate matrix inverse as opposed to operator inverse.

Whyburn [2] has shown that if we replace the boundary condition $H_{1}(Y)=D$ with the more general

$$
\sum_{d_{i} \in q} A_{i} Y\left(d_{i}\right)+\int_{a}^{b} F_{1}(x) Y(x) d x=D_{1},
$$

where $q$ is a first species subset of $[a, b]$, then there are matrices $A, B, D$, and $F(x)$ such that the nonhomogeneous system associated with (1), (2) is equivalent to this new system.

3. Cole's Green's matrix. Cole [3] has discovered that the Green's matrix

Received by the editors July 15, 1966. 


$$
\begin{aligned}
G_{C}(x, t) & =\int_{a}^{t} U(x) H_{2}^{I}(U) d F(s) U(s) U^{I}(t), & t<x \\
& =-\int_{t}^{b} U(x) H_{2}^{I}(U) d F(s) U(s) U^{I}(t), & t>x
\end{aligned}
$$

yields a solution of

$$
\begin{aligned}
& L_{2}(Y)=Y^{\prime}(x)=A(x) Y(x)+B(x), \\
& H_{2}(Y)=\sum_{1}^{m} W_{h} Y\left(a_{h}\right)+\int_{a}^{b} W(x) Y(x) d x=D,
\end{aligned}
$$

while being completely determined by the homogeneous $L_{2}(Y)=0$, $H_{2}(Y)=0$, a different system from (1), (2). $a=a_{1}<a_{2}<\cdots<a_{m}=b$, and $F(s)$ is the sum of $F_{2}(s)=\int_{a}^{s} W(x) d x$ and the step-function $F_{1}(s)$, with $F_{1}(a)=0$ and $F_{1}\left(a_{h}^{+}\right)-F_{1}\left(a_{h}^{-}\right)=W_{h}$.

The reader will not find it unduly difficult to show that

$$
\begin{aligned}
G_{C}(x, t) & =U(x) H_{2}^{I}(U)\left[\sum_{1}^{p} W_{i} U\left(a_{i}\right)+\int_{a}^{t} W(s) U(s) d s\right] U^{I}(t), \quad t<x, \\
& =-U(x) H_{2}^{I}(U)\left[\sum_{p+1}^{m} W_{i} U\left(a_{i}\right)+\int_{t}^{b} W(s) U(s) d s\right] U^{I}(t),
\end{aligned}
$$

where $a_{p} \leqq t \leqq a_{p+\mathbf{1}}$.

$t>x$,

-4. Careful examination of Whyburn's results [2] reveals that for each system (4), (5) there are several equivalent systems of the type $L_{1}(Y)=Q(x), H_{1}(Y)=D$. One of particular interest is found by defining $R(x)=\sum_{1}^{j} W_{i} A(x)$ on $a_{j}<x \leqq a_{j+1}$, so that

$$
\begin{aligned}
\int_{a}^{b} R(x) Y(x) d x= & \sum_{j=1}^{m-1} \sum_{i=1}^{j} W_{i} \int_{a_{j}}^{a_{j+1}}\left[Y^{\prime}(x)-B(x)\right] d x \\
= & \sum_{j=1}^{m-1} \sum_{i=1}^{j} W_{i}\left[Y\left(a_{j+1}\right)-Y\left(a_{j}\right)\right] \\
& -\sum_{j=1}^{m-1} \sum_{i=1}^{j} W_{i} \int_{a_{j}}^{a_{j+1}} B(x) d x \\
= & -\sum_{1}^{m} W_{i} Y\left(a_{i}\right)+\sum_{1}^{m} W_{i} Y(b) \\
& -\sum_{j=1}^{m-1} \sum_{i=1}^{j} W_{i} \int_{a_{j}}^{a_{j+1}} B(x) d x .
\end{aligned}
$$


System (4), (5) is now seen to be equivalent to the system which results from replacing the boundary condition $H_{1}(Y)=D$ by

$$
\begin{aligned}
H_{3}(Y)= & \sum_{1}^{m} W_{i} Y(b)+\int_{a}^{b}[W(x)-R(x)] Y(x) d x=D \\
& +\sum_{j=1}^{m-1} \sum_{i=1}^{j} W_{i} \int_{a_{j}}^{a_{j+1}} B(x) d x,
\end{aligned}
$$

which is a special case of Whyburn's endpoint-integral condition.

It can be shown that Whyburn's Green's matrix for $L_{1}(Y)=0$, $H_{3}(Y)=0$ is given by

$$
\begin{aligned}
G_{W}(x, t)=U(x) H_{2}^{I}(U)\left[\int_{a}^{t} W(x) U(x) d x\right. & +\sum_{1}^{p} W_{i} U\left(a_{i}\right) \\
& \left.-\sum_{1}^{p} W_{i} U(t)\right] U^{I}(t), \quad t<x, \\
= & -U(x) H_{2}^{I}(U)\left[\int_{t}^{b} W(x) U(x) d x+\sum_{p+1}^{m} W_{i} U\left(a_{i}\right)\right. \\
& \left.+\sum_{1}^{p} W_{i} U(t)\right] U^{I}(t), \quad t>x,
\end{aligned}
$$

which, since (1), (6) and (4), (5) are equivalent, also provides the solution of (4), (5).

It is noteworthy that $G_{W}$ and $G_{C}$ share the discontinuity

$$
G\left(x, x^{-}\right)-G\left(x, x^{+}\right)=E
$$

along the line $x=t$, and, while $G_{W}$ is otherwise continuous on $[a, b]$ $\times[a, b], G_{C}$ has discontinuities along the lines $t=a_{1}, \cdots, a_{m}$, given by $G_{C}\left(x, a_{h}^{+}\right)-G_{C}\left(x, a_{h}^{-}\right)=U(x) H_{2}^{I}(U) W_{h}$, where we in terpret $G_{C}\left(x, a_{1}^{-}\right)$ and $G_{C}\left(x, a_{m}^{+}\right)$as 0 . The terms $\pm \sum_{1}^{p} W_{i} U(t)$, which in $G_{W}$ absorb those latter mentioned discontinuities of $G_{C}$, are readily seen to be the only actual difference between $G_{W}$ and $G_{C}$.

5. The difference system. Several authors, including Whyburn [4], have given Green's matrices which yield the solution of the difference system

$$
\begin{aligned}
\left(Y_{i+1}-Y_{i}\right) /\left(x_{i+1}-x_{i}\right) & =R_{i} Y_{i}+S_{i}, \quad i=1,2, \cdots, m-1, \\
A Y_{0}+B Y_{m} & =C .
\end{aligned}
$$

A boundary condition more nearly analogous to $H_{1}(Y)=D$ or (5) is 


$$
H_{3}(Y)=\sum_{0}^{m} A_{j} Y_{j}=C .
$$

The system (7), (9) is more complex, and we now offer a theorem which will bring the powerful theory of Whyburn (Cole) to bear on this system.

Suppose that for $0 \leqq z_{i} \leqq x_{i+1}-x_{i}$, the matrix $z_{i} R_{i}+E$ is nonsingular. Let $U_{0}=E$ and for $i>0$,

$$
U_{i}=\prod_{j=i-1}^{0}\left[\left(x_{j+1}-x_{j}\right) R_{j}+E\right],
$$

and assume $\sum_{0}^{m} A_{j} U_{j}$ is nonsingular (necessary and sufficient for the uniqueness of solution of (7), (9)).

Theorem. If $P^{*}(x)=-R_{i}\left[\left(x-x_{i}\right) R_{i}+E\right]^{I}$ and

$$
Q^{*}(x)=-R_{i}\left[\left(x-x_{i}\right) R_{i}+E\right]^{I}\left(x-x_{i}\right) S_{i}+S_{i}
$$

for $x_{i} \leqq x \leqq x_{i+1}$, then the Whyburn's Green's matrix for

$$
\begin{aligned}
& Y^{* \prime}(x)+P^{*}(x) Y^{*}(x)=0, \\
& H^{*}\left(Y^{*}\right)=\sum_{0}^{m} A_{j} Y^{*}\left(x_{j}\right)=0
\end{aligned}
$$

yields the unique solution $Y^{*}$ of

$$
\begin{gathered}
Y^{* \prime}(x)+P^{*}(x) Y^{*}(x)=Q^{*}(x), \\
H^{*}\left(Y^{*}\right)=\sum_{0}^{m} A_{j} Y^{*}(x)=C
\end{gathered}
$$

and $Y_{i}=Y^{*}\left(x_{i}\right)$ is the unique solution of (7), (9).

REMARK. The requirement that $z_{i} R_{i}+E$ be nonsingular is not so restrictive as it may appear. If, for example, (7), (9) is an approximation to a system such as (1), (2), with (after Whyburn [4])

$$
R_{i}=\int_{x_{i}}^{x_{i+1}} P(x) d x /\left(x_{i+1}-x_{i}\right)
$$

then the required nonsingularity is automatic for sufficiently fine subdivision of $[a, b]$.

Proof of TheOREM. Let

$$
\begin{aligned}
Y^{*}(x) & =Y_{i}+\left[Y_{i+1}-Y_{i}\right]\left(x-x_{i}\right) /\left(x_{i+1}-x_{i}\right) \\
& =\left[\left(x-x_{i}\right) R_{i}+E\right] Y_{i}+\left(x-x_{i}\right) S_{i}
\end{aligned}
$$


so that $Y^{* \prime}(x)=R_{i} Y_{i}+S_{i}$ on $x_{i} \leqq x<x_{i+1}$. Solving for $Y_{i}$, we get

$$
Y_{\boldsymbol{i}}=\left[\left(x-x_{i}\right) R_{i}+E\right]^{I} Y^{*}(x)-\left[\left(x-x_{i}\right) R_{i}+E\right]^{I}\left(x-x_{i}\right) S_{i}
$$

so that

$$
\begin{aligned}
Y^{* \prime}(x)= & R_{i}\left[\left(x-x_{i}\right) R_{i}+E\right]^{I} Y^{*}(x) \\
& -R_{i}\left[\left(x-x_{i}\right) R_{i}+E\right]^{I}\left(x-x_{i}\right) S_{i}+S_{i},
\end{aligned}
$$

which is to say that $Y^{* \prime}+P^{*} Y^{*}=Q^{*}$.

If

$$
U^{*}(x)=\left[\left(x-x_{i}\right) R_{i}+E\right] \prod_{j=i-1}^{0}\left[R_{j}\left(x_{j+1}-x_{j}\right)+E\right]
$$

on $x_{i} \leqq x<x_{i+1}$, then $U^{* \prime}(x)+P^{*}(x) U^{*}(x)=0$ and $U^{*}$ is nonsingular an $[a, b]$, and $U^{*}\left(x_{i}\right)=U_{i}$ so that $\operatorname{det}\left[H^{*}\left(U^{*}\right)\right]=\operatorname{det}\left[\sum_{0}^{m} A_{j} U_{j}\right] \neq 0$ which implies the incompatibility of the homogeneous system, and is sufficient for the existence of Whyburn's Green's matrix for (10), (11).

One easily verifies that $Y_{i}=Y^{*}\left(x_{i}\right)$ is the required solution of $(7)$, (9), and this completes the proof.

REMARK. One can show that if $x_{s} \leqq t<x_{s+1}$ and $x_{i} \leqq x<x_{i+1}$, then

$$
\begin{aligned}
G_{W}(x, t)= & {\left[\left(x-x_{i}\right) R_{i}+E\right] G_{i s}\left[\left(x_{s+1}-x_{s}\right) R_{s}+E\right]\left[\left(t-x_{s}\right) R_{s}+E\right]^{I} } \\
& -\left[\left(x-x_{i}\right) R_{i}+E\right] U_{i} H_{3}^{I}(U) \sum_{0}^{s} A_{j},
\end{aligned}
$$

(where $G_{i s}$ is a difference Green's matrix yielding solution of (7), (9)) holds for all $(x, t)$ except on the interiors of triangles with vertices of the form $\left\{\left(x_{i}, x_{i}\right),\left(x_{i+1}, x_{i+1}\right),\left(x_{i+1}, x_{i}\right)\right\}$.

The author regrets that he is able to establish the above assertion only after a laborious computation, which does not seem appropriate for inclusion here. The above-mentioned Green's matrix is

$$
\begin{aligned}
G_{i s}= & \sum_{j=0}^{s} U_{i} H_{3}^{I}(U) A_{j} U_{j} U_{s+1}^{I}, & i>s, \\
& -\sum_{j=s+1}^{m} U_{i} H_{3}^{I}(U) A_{j} U_{j} U_{s+1}^{I}, & i \leqq s,
\end{aligned}
$$

and it is this writer's intention to make known certain interesting properties of this matrix at a later date.

\section{BiBLIOGRAPHY}

1. W. M. Whyburn, Differential equations with general boundary conditions, Bull. Amer. Math. Soc. 48 (1942), 692-704. 
2. - Differential systems with general boundary conditions, Seminar Reports in Mathematics, University of California Publications in Mathematics 2 (1944), $45-61$.

3. R. H. Cole, General boundary conditions for an ordinary linear differential system, Trans. Amer. Math. Soc. 111 (1964), 521-550.

4. W. M. Whyburn, On related difference and differential systems, Amer. J. Math. 51 (1929), 265-286.

The Virginia Military Institute

\section{THE ASYMPTOTIC BEHAVIOR OF A CLASS OF NONLINEAR DIFFERENTIAL EQUATIONS ${ }^{1}$}

\section{DONALD S. COHEN}

We shall study the asymptotic behavior for $t \rightarrow \infty$ of solutions of the following nonlinear differential equation:

$$
u^{\prime \prime}+f(t, u)=0 \text {. }
$$

We suppose that $f(t, u)$ satisfies the following conditions:

$\mathrm{H}-1: f(t, u)$ is continuous in $D: t \geqq 0,-\infty<u<\infty$.

H-2: The derivative $f_{u}$ exists on $D$ and satisfies $f_{u}(t, u)>0$ on $D$. H-3: $|f(t, u(t))| \leqq f_{u}(t, 0)|u(t)|$ on $D$.

An important class of functions $f(t, u)$ which satisfy conditions $\mathrm{H}-1,2,3$ is the class of twice continuously differentiable functions $f(t, u)$ which are odd and strictly monotone in $u$ with $f_{u u} \geqq 0$ for $u<0$ and $f_{u u} \leqq 0$ for $u>0$. Nonlinear eigenvalue problems involving this class of functions have been studied extensively by G. H. Pimbley [1].

For the case $f(t, u)= \pm t^{\sigma} u^{n}, \mathrm{R}$. Bellman [2] has given an exhaustive treatment of the asymptotic behavior of proper solutions (i.e., solutions which exist and have continuous derivatives for $\left.t \geqq t_{0}\right)$. For the case $f(t, u)=a(t) u^{2 n+1}$ several results on asymptotic behavior exist depending on properties of $a(t)$. References can be found in the papers of P. Waltman [3] and R. A. Moore and Z. Nehari [4].

Our basic result is that there exist solutions of (1) which approach those of $u^{\prime \prime}=0$. More precisely, we prove the

Received by the editors October 5, 1966.

1 This work was supported by the National Science Foundation under Grant No. GP-4597 at the California Institute of Technology. 